\title{
TENSOR PRODUCTS OF COMPLEMENTARY SERIES OF RANK ONE LIE GROUPS
}

\author{
GENKAI ZHANG
}

\begin{abstract}
We consider the tensor product $\pi_{\alpha} \otimes \pi_{\beta}$ of complementary series representations $\pi_{\alpha}$ and $\pi_{\beta}$ of classical rank one groups $S O_{0}(n, 1), S U(n, 1)$ and $S p(n, 1)$. We prove that there is a discrete component $\pi_{\alpha+\beta}$ for small parameters $\alpha, \beta$ (in our parametrization). We prove further that for $S O_{o}(n, 1)$ there are finitely many complementary series of the form $\pi_{\alpha+\beta+2 j}, j=0,1, \cdots, k$, appearing in the tensor product $\pi_{\alpha} \otimes \pi_{\beta}$ of two complementary series $\pi_{\alpha}$ and $\pi_{\beta}$, where $k=k(\alpha, \beta, n)$ depends on $\alpha, \beta, n$.
\end{abstract}

\section{INTRODUCTION}

The question of finding discrete components of a unitary representation $(\tau, L)$ of a Lie group $L$ under a Lie subgroup $G \subset L$ is one of the central topics in representation theory. As a problem in harmonic analysis this would involve some explicit realizations of representations for $L$ and $G$, the existence and boundedness of intertwining operators from $(\tau, L)$ to discrete components $(\pi, G)$. The problem of finding formal intertwining operators from the smooth representations $\left(\tau^{\infty}, L\right)$ to $\left(\pi^{\infty}, G\right)$ can be studied somewhat by algebraic methods. Given the intertwining operators the next question is to prove their boundedness. In the present paper we shall consider the case when $\left(\pi_{\gamma}, G\right)$ is a complementary series of a classical rank one group $G$ and $(\tau, L)=\left(\pi_{\alpha} \otimes \pi_{\beta}, G \times G\right)$ is a tensor product of the same type. We prove the existence of discrete components $\left(\pi_{\gamma}, G\right)$ in the tensor product.

The complementary series are unitarization of principal series and can be realized as function spaces on the Euclidean space $\mathbb{R}^{n-1}$ or $H$-type groups. There exist certain bilinear invariant differential intertwining operators on the tensor products, and the study of them is of interesting for various purposes. The most well-known examples of similar operators might be the Rankin-Cohen brackets on tensor products of holomorphic discrete series of $\mathrm{SL}(2, \mathbb{R})$, which yield also a decomposition of the tensor products in the unitary sense. In [21] Ovsienko and Redou have found a family of formal differential operators on tensor product of principal series $\pi_{\alpha}, \alpha \in \mathbb{C}$, of the conformal group $S O(n, 1)$. We shall prove the boundedness for the intertwining operators under certain conditions, thus proving the existence of finitely many discrete components. For the group $S U(n, 1)$ and $S p(n, 1)$ we prove the natural restriction operator is bounded and found then one discrete component, namely $\pi_{\alpha+\beta}$ in $\pi_{\alpha} \otimes \pi_{\beta}$. Our main results are stated in Theorems 4.1 and 5.1.

We give a very brief account of the closely related results. The Rankin-Cohen brackets for holomorphic representations and their generalizations have been studied in various

This research was partially supported by the Swedish Science Council (VR). 
contexts. An analytic and effective method to derive them is via the reproducing formula. The similar tools for spherical representations are the Knapp-Stein intertwining operators. The intertwining operators in [21] are found by using an Ansatz expressing the operators as polynomials of the Laplacian operators $\mathcal{L}_{x}, \mathcal{L}_{y}$, and the inner product $\nabla_{x} \cdot \nabla_{y}$ evaluated on the diagonal $x=y$. The same operators are obtained in [5] as residues of a family of integral bilinear intertwining operators, which is based on some earlier works on trilinear form [4, 6, 11, 2], which are variations of the Knapp-Stein intertwining operator. Kobayashi and Pevzner [16] have constructed systematically invariant bilinear differential operators using special orthogonal polynomials. Tensor product decompositions for representations of $S L(2, \mathbb{R})$ had been studied in greater details in the work of Pukanszky [23] and by Repka [24]; see also [1]. Theses results combined with the general theory of Burger-Li-Sarnak [3] have also found applications in automorphic forms [7]. Tensor products of representations of $S L(2, \mathbb{C})$ (i.e., locally isomorphic to the Lorentz group $S O_{0}(3,1)$ ) have been studied by Naimark [19]; see also [20] where some complementary series representations were constructed using restriction of holomorphic representations, the same idea being used in the present paper in the construction of discrete components for the groups $S U(n, 1)$ and $S p(n, 1)$. A related question is the branching of complementary series of rank one groups $L=S O(n, 1), S U(n, 1), S p(n, 1)$ under the subgroups $G=S O(n-1,1), S U(n, 1)$, $S p(n, 1)$ and is studied in [18, 27, 29].

The paper is organized as follows. In Section 2 we recall the realization of the complementary series of $S O(n, 1)$ as function spaces on $\mathbb{R}^{n-1}$. For the groups $G=S U(n, 1)$ and $S p(n, 1)$ we shall need their realization in terms of holomorphic representations of some Hermitian groups containing $G$. In Section 3 we present an elementary and independent construction of the bilinear differential operators for $S O(n, 1)$. While the technical computations are slightly different from those in the existing literature, the underlying idea is still the same, the explicit application of Knapp-Stein operators here making the computations conceptually clear. Section 4 is devoted to the proof of the appearance of finitely many discrete components, $\pi_{\alpha+\beta+2 j}, j \geq 0$, in the tensor product $\pi_{\alpha} \otimes \pi_{\beta}, \alpha, \beta>0$, when the parameters $\alpha$ and $\beta$ are relatively small (in our parametrization); In $\S 5$ we treat the other rank one groups $S U(n, 1)$ and $S p(n, 1)$.

It is clear now that there remain many interesting problems in understanding the discrete components of the tensor products of complementary series, in particular for the other rank one groups $S U(n, 1), S p(n, 1)$ and exceptional $F_{4}$-group. The spherical representations are then realized on H-type groups and the Euclidean Fourier analysis in Section 4 might have to be replaced by Fourier analysis on the nilpotent groups. First of all it would be interesting to construct bilinear invariant differential operators. Secondly one may proceed to prove the boundedness and it seems estimating these operators becomes far more complicated. On the hand one might also try the method of holomorphic extension as in the present paper.

I would like to thank Jean-Louis Clerc for some stimulating discussions. 


\section{SPHERICAL REPRESENTATIONS OF RANK ONE GROUP $G$}

We fix notation and recall some known results on induced representations of $G$ and the Knapp-Stein intertwining operator. We shall use the non-compact realization of the representations. We shall be quite brief, and most of the technical formulas can be found e.g. in [14, 9] where the general case of rank one groups is studied.

2.1. Classical rank one Lie groups. Let $G=S O_{0}(n, 1, \mathbb{F})=S O_{0}(n, 1), S U(n, 1), S p(n, 1)$, $\mathbb{F}=\mathbb{R}, \mathbb{C}, \mathbb{H}$, be the classical rank one Lie group in its standard realization [14, 27]. Let $\mathfrak{g}=\mathfrak{k}+\mathfrak{p}$ be the Cartan decomposition of the Lie algebra $\mathfrak{g}$ of $G$. We fix an element $H \in \mathfrak{p}$ and the subspace $\mathfrak{a}:=\mathbb{R} H_{0} \in \mathfrak{p}$ such that $\operatorname{Ad}(H)$ has (possible) eigenvalues $\pm 2, \pm 1,0$. The root space decomposition of $\mathfrak{g}$ under $H$ is

$$
\mathfrak{g}=\mathfrak{n}_{-2}+\mathfrak{n}_{-1}+(\mathfrak{a}+\mathfrak{m})+\mathfrak{n}_{1}+\mathfrak{n}_{2}
$$

with eigenvalues $\pm 2, \pm 1,0$ if $\mathbb{F}=\mathbb{C}, \mathbb{H}$, and $\pm 1,0$ if $\mathbb{F}=\mathbb{R}$. Here $\mathfrak{m} \subset \mathfrak{l}$ is the zero root space. We denote by $\mathfrak{n}=\mathfrak{n}_{1} \oplus \mathfrak{n}_{2}$ the sum of the positive root spaces. Then $\mathfrak{m}+\mathfrak{a}+\mathfrak{n}$ is a maximal parabolic subalgebra of $\mathfrak{g}$.

Let $\rho$ be the half sum of positive roots. Then

$$
\rho(H)= \begin{cases}\frac{n-1}{2}, & \mathbb{F}=\mathbb{R} \\ n, & \mathbb{F}=\mathbb{C} \\ 2 n+1, & \mathbb{F}=\mathbb{H}\end{cases}
$$

and we write $\rho=\rho(H)$.

2.2. Spherical representations and complementary series for $G=S O_{0}(n, 1 ; \mathbb{F})$. Denote $M, A, N$ the corresponding subgroups of $G$ with Lie algebras $\mathfrak{m}, \mathfrak{a}, \mathfrak{n}$, and $P=M A N$ the parabolic subgroup. For $\mu \in \mathbb{C}$ let $\pi_{\mu}^{\infty}$ be the induced smooth representation of $G$ from the character $e^{-\mu}: m e^{t H} n \in P=M A N \mapsto e^{-\mu t}$, consisting of $C^{\infty}$-functions $f$ on $G$ such that

$$
f\left(g m e^{t H_{0}} n\right)=e^{-\mu t} f(g), m e^{t H_{0}} n \in M A N .
$$

In particular $f$ are determined by their restriction on $K$ and are identified further as smooth functions on $K / M$. We have $\pi_{\mu}^{\infty}=C^{\infty}(K / M)=C^{\infty}(S)$ as vector spaces. Restricting smooth functions in $\pi_{\mu}^{\infty}$ to $N^{-}$results in an injective map to a subspace of $C^{\infty}\left(N^{-}\right)=$ $C^{\infty}\left(\mathbb{R}^{n-1}\right)$. We shall fix this realization of $\pi_{\mu}^{\infty}$. (Indeed it is only a proper subspace of $C^{\infty}\left(N^{-}\right)$as some matching conditions at infinity are needed.)

The explicit formulas for $\pi_{\mu}(g)$ can be found in [14] in the compact picture and in [26] for the non-compact picture. We shall only need them for the real group $G=S O_{0}(1, n)$. In this case $\ltimes=\mathbb{R}^{n-1}$ and $G$ is generated by the parabolic group $M A N^{-}$and the Weyl group element $w,\left[10\right.$, Theorem $1.4, \mathrm{Ch}$. IX], where $M A N^{-}$acts on $\mathbb{R}^{n-1}$ by the defining action and $w$ by inversion, $w(x)=-\frac{1}{x}:=-\frac{x}{|x|^{2}}$. Their actions on $\pi_{\mu}^{\infty}$ are given by $\pi_{\mu}(g) f(x)=e^{-t \mu} f\left(e^{t} m^{-1}\left(x-x_{0}\right)\right), \quad\left(m, e^{t H}, x_{0}\right)=m e^{t H} x_{0} \in M A N^{-}, \quad N^{-}=\mathbb{R}^{n-1}$, 
and

$$
\pi_{\mu}(w) f(x)=\|x\|^{-2 \mu} f\left(-\frac{x}{\|x\|^{2}}\right) .
$$

Note also that the Jacobians of $g=\left(m, e^{t H}, x_{0}\right)$ and of the Weyl group element $w$ on $N^{-}=\mathbb{R}^{n-1}$ are given by

$$
J_{g}(x)=e^{t(n-1)}, \quad J_{w}(x)=\frac{1}{|x|^{2(n-1)}} .
$$

The representation $\pi_{\mu}(g), g \in G, \mu \in(\rho+i \mathbb{R})$ is already unitary for the natural unitary norm in $L^{2}(K / M)$. However for certain real $\mu \in \mathbb{R}$ it is possible to define a different $\mathfrak{g}$-invariant inner product on the space of $K$-finite vectors, in which case its Hilbert space completion is called complementary series; see [14]. The precise range of the parameters $\mu$ is given by

$$
\mu \in\left\{\begin{array}{l}
(0,2 \rho), \mathbb{F}=\mathbb{R}, \mathbb{C} \\
(2,2 \rho-2), \mathbb{F}=\mathbb{H} .
\end{array}\right.
$$

We shall denote the corresponding representation still by $\pi_{\mu}$, and shall use its non-compact realization for the real case $G=S O_{0}(n, 1)$, allowing us to find (generically) more than one discrete components in the tensor product decomposition in $\S 4$.

2.3. Realization of complementary series for $G=S O_{0}(n, 1)$ on $\mathbb{R}^{n-1}$. The unitarization of the complementary series is obtained via the Knapp-Stein intertwining operator, defined preliminarily on $K$-finite vectors (which can be obtained from $K$-finite vectors on $K / M$ via Cayley transform),

$$
J_{\mu} f(x)=\int_{\mathbb{R}^{n-1}} K_{\mu}(x, y) f(y) d y,
$$

where

$$
K_{\mu}(x, y)=C_{\mu} \frac{1}{|x-y|^{2 \mu}}, \quad C_{\mu}=\frac{\Gamma\left(\rho-\frac{\mu}{2}\right) \Gamma\left(\rho-\frac{\mu}{2}+\frac{1}{2}\right)}{\Gamma\left(\frac{n}{2}\right) \Gamma(\rho-\mu)} .
$$

(The normalization is chosen here so that in the compact picture $J_{\mu} 1_{S}=1_{S}$, where $1_{S}$ is the constant function on $S$ viewed as a function on $G$ restricted to $\mathbb{R}^{n-1}$.)

Then $J_{\mu}$ is a $G$-intertwining operator

$$
J_{\mu}: \pi_{\widetilde{\mu}}^{\infty} \rightarrow \pi_{\mu}^{\infty}, \quad \widetilde{\mu}:=2 \rho-\mu
$$

for $\mu<<0$. It has holomorphic continuation to the whole complex plane, and in particular holomorphic and non-zero in the two symmetric strips around $\Re \mu=\rho$,

$$
\{\mu ; 0<\Re \mu<\rho\}, \quad\{\mu ; \rho<\Re \mu<2 \rho\} .
$$

The formal intertwining property can be proved by using the following transformation rule of $K_{\mu}$,

$$
K_{\mu}(g z, g w)=|(c z+d)|^{-\mu} K_{\mu}(z, w)|(c w+d)|^{-\mu}=J_{g}(z)^{\frac{\mu}{n-1}} K_{\mu}(z, w) J_{g}(w)^{\frac{\mu}{n-1}}
$$


where $J_{g}$ is the Jacobian of the action of $g \in G$ on $N^{-}=\mathbb{R}^{n-1}$. See [15, Chapter VII] and [28] for some general theory of intertwining operators.

The inner product

$$
\left(f_{1}, f_{2}\right)_{\mu}=\left(J_{\widetilde{\mu}} f_{1}, f_{2}\right)_{L^{2}\left(\mathbb{R}^{n-1}\right)}
$$

for $f_{1}, f_{2} \in C_{0}^{\infty}\left(\mathbb{R}^{n-1}\right)$ is a pre-Hilbert norm, and is invariant under $g \in G$ sufficiently close to the identity (depending on $f_{1}, f_{2}$ ). The completion defines the complementary series, $\mu \in(0,2 \rho)$. We shall use its description in term of Fourier transform $f \mapsto \mathcal{F} f$. The space $\pi_{\mu}$ is the completion of $C_{0}^{\infty}\left(\mathbb{R}^{n-1}\right)$ under the (equivalent) norm

$$
\|f\|_{\mu}^{2}=\int_{\mathbb{R}^{n-1}}|\mathcal{F} f(\xi)|^{2}|\xi|^{n-1-2 \mu} d \xi=\left\|\mathcal{F} f(\cdot)|\cdot|^{\frac{\widetilde{\mu}}{2}}\right\|_{L^{2}\left(\mathbb{R}^{n-1}\right)}^{2}
$$

for $0<\mu<2 \rho$. See e.g. [26].

2.4. Complementary series for $G=S U(n, 1), S p(n, 1)$ and their holomorphic extensions. We shall use a different method for the cases $G=S U(n, 1), S p(n, 1)$. The method is based on, roughly speaking, holomorphic extension.

We consider a Hermitian Lie group $G_{1}$ containing $G$ as a symmetric subgroup such that $G / K$ is a real form of the Hermitian subgroup $G_{1} / K_{1}$; see [12, 27]. In certain rather general context the similar groups $G_{1}$ are also called overgroups in some Russian litterature; see e. g. [17].

More precisely let

$$
G_{1}=\left\{\begin{array}{l}
S U(n, 1) \times S U(n, 1), \mathbb{F}=\mathbb{C} \\
S U(2 n, 2), \mathbb{F}=\mathbb{H},
\end{array}\right.
$$

with $G=S U(n, 1), S p(n, 1)$ being realized as the diagonal subgroup of $G_{1}$ and respectively as complex transformations via the standard identification of $\mathbb{H}=\mathbb{C}^{2}$.

The holomorphic discrete series of $G_{1}$ can be realized on the space of holomorphic functions on $D_{1}$. To fix notation we let

$$
V_{1}= \begin{cases}\mathbb{C}^{n} \oplus \overline{\mathbb{C}^{n}}, & \mathbb{F}=\mathbb{C} \\ M_{2 n, 2}(\mathbb{C}), & \mathbb{F}=\mathbb{H},\end{cases}
$$

and the space $D_{1}=G_{1} / K_{1}$ is realized as a bounded symmetric domain in $V_{1}$,

$$
D_{1}= \begin{cases}B^{n} \times \overline{B^{n}}, & \mathbb{F}=\mathbb{C} \\ \left\{Z \in M_{2 n, 2}(\mathbb{C}) ; Z^{*} Z<I\right\}, & \mathbb{F}=\mathbb{H} .\end{cases}
$$

Let $\mathcal{H}_{\nu}\left(D_{1}\right)$ be the space of holomorphic functions on $D_{1}$ with reproducing kernel $h(z, w)^{-\nu}$ for $\nu$ sufficiently large, where

$$
h(z, w)= \begin{cases}\left(1-\left\langle z_{1}, w_{1}\right\rangle\right)\left(1-\left\langle w_{2}, z_{2}\right\rangle\right), & \mathbb{F}=\mathbb{C} \\ \operatorname{det}\left(1-w^{*} z\right), & \mathbb{F}=\mathbb{H} .\end{cases}
$$


It is now well-known [13] that if $\nu$ is in the set

$$
\begin{cases}(0, \infty) & \mathbb{F}=\mathbb{R}, \mathbb{C} \\ (1, \infty) & \mathbb{F}=\mathbb{H},\end{cases}
$$

then the kernel $h(z, w)^{-\nu}$ is positive definite and it defines a unitary (projective) representation of $G_{1}$. We denote this representation by $\left(\mathcal{H}_{\nu}\left(D_{1}\right), \tau_{\nu}, G_{1}\right)$.

We recall the following theorem [12].

Theorem 2.1. The complementary series $\left(\pi_{\mu}, G\right)$ appears as a discrete summand in $\left(\mathcal{H}_{\nu}\left(D_{1}\right), \tau_{\nu}, G_{1}\right)$ restricted to $G$ if $\nu$ and $\mu$ are related by

$$
2 \nu= \begin{cases}\mu, \mu \in(0, n), & \mathbb{F}=\mathbb{C} \\ \mu, \mu \in(2,2 n-1), & \mathbb{F}=\mathbb{H} .\end{cases}
$$

Note that the range of $\mu$ is, disregarding the Weyl group symmetry, precisely the whole range of the complementary series representations. In other words, any complementary series of $G$ is a discrete component in an holomorphic representation of $G_{1}$.

\section{INVARIANT BILINEAR DIFFERENTIAL OPERATORS FOR GENERAL SPHERICAL$$
\text { SERIES REPRESENTATIONS OF } G=S O_{0}(n, 1)
$$

We denote $\pi_{\alpha}^{\infty} \otimes \pi_{\beta}^{\infty}$ the induced smooth representation of $G \times G$ from the parabolic subgroup $P \times P$; see (2.1). (The tensor notation here is a bit ambiguous, and the precise definition should be that it is the induced representation from the tensor product of representations of $P$.) The group $G$ is viewed as the diagonal subgroup of $G \times G$.

Theorem 3.1. For any nonnegative integer $m \geq 0$ there exists a $G$-intertwining differential operator $\mathcal{D}_{\alpha, \beta, m}$ of degree $2 m$ meromorphic in $(\alpha, \beta) \in \mathbb{C}^{2}$,

$$
\mathcal{D}_{\alpha, \beta, m}: \pi_{\alpha}^{\infty} \otimes \pi_{\beta}^{\infty} \rightarrow \pi_{\alpha+\beta+2 m}^{\infty}
$$

The only possible poles of $\mathcal{D}_{\alpha, \beta, m}$ appear when $\alpha$ or $\beta \in \Lambda_{m}$, where

$$
\Lambda_{j}=\{0,-1, \cdots,-m+1\} \cup(\rho-1+\{0,-1, \cdots,-m+2\}) .
$$

The proof will be divided into a few elementary steps.

Let $S_{\alpha, \beta, m}(x, y ; z, w)$ be the kernel

$$
S_{\alpha, \beta, m}(x, y ; z, w)=\left(\frac{|(x-z)-(y-w)|^{2}}{|x-z|^{2}|y-w|^{2}}\right)^{m} \frac{1}{|x-z|^{2 \alpha}|y-w|^{2 \beta}},
$$

and write for simplicity

$$
S_{\alpha, \beta, m}(x ; z, w)=S_{\alpha, \beta, m}(x, x ; z, w),
$$

the restriction to the diagonal $x=y$ of $S_{\alpha, \beta, m}(x, x ; z, w)$. 
Lemma 3.2. The integral operator

$$
T_{m} f(x)=T_{\alpha, \beta, m} f(x):=C_{\alpha} C_{\beta} \int_{\mathbb{R}^{2(n-1)}} S_{\alpha, \beta, m}(x ; z, w) f(z, w) d z d w
$$

defines an intertwining operator

$$
\pi_{\widetilde{\alpha}}^{\infty} \otimes \pi_{\widetilde{\beta}}^{\infty} \rightarrow \pi_{\alpha+\beta+2 m}^{\infty}
$$

for $\alpha, \beta \in \mathbb{R}$ sufficiently negative and has meromorphic continuation to all $\alpha, \beta \in \mathbb{C}$.

Proof. Recall the group $G$ is generated by $P$ and $w$. The formal intertwining property follows directly from a change of variables $(x, y) \mapsto(g x, g y)$ for $g \in P$ and $g=w$ along with the formula (2.2) for the Jacobians. To prove the meromorphic continuation in $\alpha$ and $\beta$ we observe that changing $(x, y)$ to $(x-z, y-z)$ we need only to prove that the integral

$$
\int_{\mathbb{R}^{2(n-1)}} \frac{|x-y|^{2 m}}{|x|^{2 m}|y|^{2 m}} \frac{1}{|x|^{2 \alpha}|y|^{2 \beta}} f(x, y) d x d y
$$

is meromorphic in $(\alpha, \beta)$. But this is just up to normalization constants the integral $\left(J_{\alpha+m} \otimes\right.$ $\left.J_{\beta+m}\right)(F), F(x, y)=|x-y|^{2 m} f(x, y)$ and thus has the continuation.

In the compact-realization this operator is

$$
T_{m} f(x)=C_{\alpha} C_{\beta} \int_{S \times S} \frac{(1-\langle z, w\rangle)^{m}}{(1-\langle x, z\rangle)^{m+\alpha}(1-\langle x, w\rangle)^{m+\beta}} f(z, w) d z d w .
$$

That the integral is well-defined for $\alpha, \beta<<0$ can also be deduced from this formula.

Next we need some elementary known identities for the Laplacian operator $\mathcal{L}=\partial_{1}^{2}+$ $\cdots+\partial_{n}^{2}$ acting on $|x|^{-2 \alpha}$, the so-called Bernstein-Sato identities. Recall the Pochammer symbol defined by $(\alpha)_{j}=\alpha(\alpha+1) \cdots(\alpha+j-1)$.

Lemma 3.3. The following differentiation formula holds

$$
\mathcal{L}^{j}|x|^{-2 \alpha}=2^{2 j}(\alpha)_{j}(\alpha+1-\rho)_{j}|x|^{-2(\alpha+j)}, \quad x \neq 0 .
$$

We define inductively a family of differential operators of constant coefficients on $C^{\infty}\left(\mathbb{R}^{2(n-1)}\right)$ by

$$
M_{\alpha, \beta, 0}=I, \quad M_{\alpha, \beta, 1}=\nabla_{x} \cdot \nabla_{y}
$$

and

$$
\mathcal{M}_{\alpha, \beta, j+1}=\left(\nabla_{x} \cdot \nabla_{y}\right) \mathcal{M}_{\alpha, \beta, j}-\frac{j(n-1-3 j-2 \alpha-2 \beta)}{(\alpha+1-\rho)(\beta+1-\rho)} \mathcal{M}_{\alpha+1, \beta+1, j-1} \mathcal{L}_{x} \mathcal{L}_{y}
$$

It follows from the construction that the only possible poles of $M_{\alpha, \beta, j}, j \geq 2$, appear when $\alpha$ or $\beta$ is in

$$
\{\rho-i ; i=1, \cdots, j-1\} .
$$

Lemma 3.4. The following formula holds for all $(\alpha, \beta) \in \mathbb{C}^{2}$ and $m=0,1,2, \cdots$,

$$
\mathcal{M}_{\alpha, \beta, m}\left(\frac{1}{|x|^{2 \alpha}|y|^{2 \beta}}\right)=2^{2 j}(\alpha)_{m}(\beta)_{m}\left(\frac{\langle x, y\rangle}{|x|^{2}|y|^{2}}\right)^{m}\left(\frac{1}{|x|^{2 \alpha}|y|^{2 \beta}}\right) \text {. }
$$


Proof. We write the identify as $\mathrm{LHS}_{m}=\mathrm{RHS}_{m}$ prove it by induction on $m$.

The identity is trivially true for $m=0$. Assuming the identity holds for $0 \leq m \leq j$ for all $\alpha, \beta$. We perform the differentiation. $\nabla_{x} \cdot \nabla_{y}$ on the identity with $m=j$ and find

$$
\nabla_{x} \cdot \nabla_{y} \mathcal{M}_{\alpha, \beta, j} \frac{1}{|x|^{2 \alpha}|y|^{2 \beta}}=2^{2 j}(\alpha)_{j}(\beta)_{j}(I+I I)=2^{2 j}(\alpha)_{j}(\beta)_{j} I+2^{2 j}(\alpha)_{j}(\beta)_{j} I I
$$

a sum of two terms, with the first term

$$
\begin{aligned}
2^{2 j}(\alpha)_{j}(\beta)_{j} I & =2^{2 j}(\alpha)_{j}(\beta)_{j} 2^{2}(\alpha+j)(\beta+j)\left(\frac{\langle x, y\rangle}{|x|^{2}|y|^{2}}\right)^{j+1} \frac{1}{|x|^{2 \alpha}|y|^{2 \beta}} \\
& =2^{2(j+1)}(\alpha)_{j+1}(\beta)_{j+1}\left(\frac{\langle x, y\rangle}{|x|^{2}|y|^{2}}\right)^{j+1}\left(\frac{1}{|x|^{2 \alpha}|y|^{2 \beta}}\right)=\text { RHS }_{j+1}
\end{aligned}
$$

the the RHS of (3.4) for $m=j+1$, and

$$
I I=j(n-1-3 j-2 \alpha-2 \beta)\left(\frac{\langle x, y\rangle}{|x|^{2}|y|^{2}}\right)^{j-1}\left(\frac{1}{|x|^{2(\alpha+1)}|y|^{2(\beta+1)}}\right) .
$$

We treat the second term using the induction hypothesis for $m=j-1$ with $(\alpha, \beta)$ being replaced by $(\alpha+1, \beta+1)$,

$$
\begin{aligned}
& 2^{2(j-1)}(\alpha+1)_{j-1}(\beta+1)_{j-1}\left(\frac{\langle x, y\rangle}{|x|^{2}|y|^{2}}\right)^{j-1}\left(\frac{1}{|x|^{2(\alpha+1)}|y|^{2(\beta+1)}}\right) \\
= & \mathcal{M}_{\alpha+1, \beta+1, j-1}\left(\frac{1}{|x|^{2(\alpha+1)}|y|^{2(\beta+1)}}\right)
\end{aligned}
$$

which is furthermore

$$
\frac{1}{2^{2} \alpha(\alpha+\rho-1) \beta(\beta+\rho-1)} \mathcal{M}_{\alpha+1, \beta+1, j-1} \mathcal{L}_{x} \mathcal{L}_{y}\left(\frac{1}{|x|^{2(\alpha)}|y|^{2(\beta)}}\right) .
$$

We rewrite (3.5) as

$$
\begin{aligned}
\operatorname{RHS}_{j+1} & =\left(\nabla_{x} \cdot \nabla_{y} \mathcal{M}_{\alpha, \beta, j}-\frac{j(n-1-3 j-2 \alpha-2 \beta)}{(\alpha+\rho-1)(\beta+\rho-1)} \mathcal{M}_{\alpha+1, \beta+1, j-1} \mathcal{L}_{x} \mathcal{L}_{y}\right) \frac{1}{|x|^{2(\alpha)}|y|^{2(\beta)}} \\
& =\mathcal{M}_{\alpha, \beta, j+1}\left(\frac{1}{|x|^{2(\alpha)}|y|^{2(\beta)}}\right) \\
& =\operatorname{LHS}_{j+1}
\end{aligned}
$$

by the definition. This finishes the proof.

Combining the two Lemmas we have

$$
\mathcal{M}_{\alpha+j, \beta+i, k} \mathcal{L}_{x}^{j} \mathcal{L}_{x}^{i} \frac{1}{|x|^{2 \alpha}|y|^{2 \beta}}=c_{i, j, k}(\alpha, \beta)\left(\frac{\langle x, y\rangle}{|x|^{2}|y|^{2}}\right)^{k} \frac{1}{|x|^{2 \alpha+2 j}|y|^{2 \beta+2 i}},
$$

where

$$
c_{i, j, k}(\alpha, \beta)=2^{2 k+2 j+2 i}(\alpha)_{j+k}(\alpha+1-\rho)_{j}(\beta)_{i+k}(\beta+1-\rho)_{i} .
$$

Here we have used the fact that

$$
(\gamma)_{j}(\gamma+j)_{k}=(\gamma)_{j+k}
$$


By translation invariance we have

$$
\begin{aligned}
& \mathcal{M}_{\alpha+j, \beta+i, k} \mathcal{L}_{x}^{j} \mathcal{L}_{x}^{i} \frac{1}{|x-z|^{2 \alpha}|y-w|^{2 \beta}} \\
= & c_{i, j, k}(\alpha, \beta)\left(\frac{\langle x-z, y-w\rangle}{|x-z|^{2}|y-w|^{2}}\right)^{k} \frac{1}{|x-z|^{2 \alpha+2 j}|y-w|^{2 \beta+2 i}},
\end{aligned}
$$

We prove now Theorem 3.1.

Proof. The operator

$$
T_{\alpha, \beta, m}\left(J_{\alpha} \otimes J_{\beta}\right): \pi_{\widetilde{\alpha}}^{\infty} \otimes \pi_{\widetilde{\beta}}^{\infty} \rightarrow \pi_{\alpha+\beta+2 m}^{\infty}
$$

is an intertwining operator by Lemma 3.2. We prove it is a differential operator. The idea is to differentiate the identity $f=\left(J_{\alpha} \otimes J_{\beta}\right)\left(J_{\widetilde{\alpha}} \otimes J_{\widetilde{\beta}}\right) f$. We shall perform formal computations on the integral first and justify the procedure in the end. Let $f \in \pi_{\alpha}^{\infty} \otimes \pi_{\beta}^{\infty}$ and $g=\left(J_{\widetilde{\alpha}} \otimes J_{\widetilde{\beta}}\right) f$. We denote

$$
\begin{aligned}
& \mathcal{E}_{\alpha, \beta, m} f(x, y) \\
= & \sum_{i+j+k=m} \varepsilon_{i, j, k}(\alpha, \beta) \mathcal{M}_{\alpha+j, \beta+i, k} \mathcal{L}_{x}^{j} \mathcal{L}_{y}^{i} f(x, y)
\end{aligned}
$$

and

$$
\mathcal{D}_{\alpha, \beta, m} f(x)=\left.\mathcal{E}_{\alpha, \beta, m} f\right|_{x=y},
$$

for $f \in C^{\infty}\left(\mathbb{R}^{2(n-1)}\right)$, where

$$
\varepsilon_{i, j, k}(\alpha, \beta):=\left(\begin{array}{c}
m \\
i, j, k
\end{array}\right) \frac{(-2)^{k}}{c_{i, j, k}(\alpha, \beta)} .
$$

We claim that

$$
\mathcal{D}_{\alpha, \beta, m} f=T_{\alpha, \beta, m}\left(J_{\alpha} \otimes J_{\beta}\right) f, \quad f \in \pi_{\widetilde{\alpha}}^{\infty} \otimes \pi_{\widetilde{\beta}}^{\infty}
$$

proving the formal intertwining property of the differential operator $\mathcal{D}_{\alpha, \beta, m}$.

The binomial expansion of $S(x, y ; z, w)$ reads as follows

$$
\begin{aligned}
S(x, y ; z, w) & =\left(\frac{|x-z|^{2}+|y-w|^{2}-2\langle x-z, y-w\rangle}{|x-z|^{2}|y-w|^{2}}\right)^{m} \frac{1}{|x-z|^{2 \alpha}|y-w|^{2 \beta}} \\
& =\sum_{i+j+k=m}\left(\begin{array}{c}
m \\
i, j, k
\end{array}\right)(-2)^{k}\left(\frac{\langle x-z, y-w\rangle}{|x-z|^{2}|y-w|^{2}}\right)^{k} \frac{1}{|x-z|^{2 j+2 \alpha}|y-w|^{2 i+2 \beta}} .
\end{aligned}
$$

Summing the formula (3.7) over $(i, j, k)$ we have then

$$
\mathcal{E}_{\alpha, \beta, m} \frac{1}{|x-z|^{2 \alpha}|y-w|^{2 \beta}}=S_{\alpha, \beta, m}(x, y ; z, w)
$$

which further implies that

$$
\mathcal{D}_{\alpha, \beta, m}\left(\frac{1}{|x-z|^{2 \alpha}|y-w|^{2 \beta}}\right)=S_{\alpha, \beta, m}(x, x ; z, w)=S_{\alpha, \beta, m}(x ; z, w) .
$$


The identity $f=\left(J_{\alpha} \otimes J_{\beta}\right)\left(J_{\widetilde{\alpha}} \otimes J_{\widetilde{\beta}}\right) f=\left(J_{\alpha} \otimes J_{\beta}\right) g$ reads

$$
f(x, y)=\left(J_{\alpha} \otimes J_{\beta}\right) g=C_{\alpha} C_{\beta} \int_{\mathbb{R}^{2(n-1)}} \frac{1}{|x-z|^{2 \alpha}|y-w|^{2 \beta}} g(z, w) d z d w .
$$

We perform the differentiation $\mathcal{D}_{\alpha, \beta, m}$ on this identity and find

$$
\mathcal{D}_{\alpha, \beta, m} f(x)=C_{\alpha} C_{\beta} \int_{\mathbb{R}^{2(n-1)}} S_{\alpha, \beta, m}(x ; z, w) g(z, w) d z d w=T_{m} g(x)=T_{m} J_{\widetilde{\alpha}} \otimes J_{\widetilde{\beta}} f(x),
$$

proving (3.9).

Finally the differentiation under integral sign can be justified by taking first $\alpha, \beta<<0$ and $\alpha \notin \mathbb{Z}_{-}, \beta \notin \mathbb{Z}_{-}$, with $\widetilde{\alpha}>>0, \widetilde{\beta}>>0$, in which case Lemma 2.1 implies that all integrals involved are absolutely convergent. The meromorphic continuation is proved in Lemma 3.2 and the possible poles are read off from the definition of $\mathcal{D}_{\alpha, \beta, m}$ and $\mathcal{M}_{\alpha, \beta, m}$ in (3.8) respectively (3.3). This finishes the proof.

\section{Finitely many discrete COMPONENTS IN THE TENSOR PRODUCT $\pi_{\alpha} \otimes \pi_{\beta}$,

$$
G=S O_{o}(n, 1, \mathbb{R})
$$

We apply the intertwining operators $\mathcal{D}_{m}=\mathcal{D}_{\alpha, \beta, m}$ to the study of appearance of discrete components in the tensor product $\pi_{\alpha} \otimes \pi_{\beta}$ of complementary series. For $\alpha, \beta \in(0, \rho)$ the tensor product $\pi_{\alpha} \otimes \pi_{\beta}$ in the non-compact picture is the completion of $C_{0}^{\infty}\left(\mathbb{R}^{2(n-1)}\right)$ with norm

$$
\|f\|_{\alpha \otimes \beta}^{2}:=\int_{\mathbb{R}^{2(n-1)}}|\mathcal{F} f(\xi, \eta)|^{2}|\xi|^{n-2 \alpha}|\eta|^{n-2 \beta} d \xi d \eta
$$

cf. (2.8).

Theorem 4.1. Suppose $0<\alpha, \beta<\rho$ and $m \geq 0$ are integers. If $\alpha+\beta+2 m<\rho$ then the intertwining operator $\mathcal{D}_{\alpha, \beta, m}$ is a non-zero bounded intertwining operator $\pi_{\alpha} \otimes \pi_{\beta} \rightarrow$ $\pi_{\alpha+\beta+2 m}$. Thus there are $k$ discrete components $\pi_{\alpha+\beta+2 m}$ appears in the tensor product $\pi_{\alpha} \otimes \pi_{\beta}$ for $m=0,1, \cdots, k$ where $k$ is the maximal integer such that $\alpha+\beta+2 k<\rho$.

Proof. Noticing that for $\alpha, \beta$ and $m$ as above we have that the operator $\mathcal{D}_{m}=\mathcal{D}_{\alpha, \beta, m}$ is well-defined on smooth functions, and $\pi_{\alpha}, \pi_{\beta}$ and $\pi_{\alpha+\beta+2 m}$ are unitary representations. Recall also the notation $\tilde{\alpha}=2 \rho-\alpha=n-1-\alpha$ in $\S 2.3$ and the unitary norm (2.8). Let $f \in C_{0}^{\infty}\left(\mathbb{R}^{2(n-1)}\right) \subset \pi_{\alpha} \otimes \pi_{\beta}$. We claim that

$$
\left\|\mathcal{D}_{m} f\right\|_{\alpha+\beta+2 m}^{2} \leq C\|f\|_{\alpha \otimes \beta}^{2}
$$

Thus $\mathcal{D}_{m}$ defines a non-zero intertwining operator from $\pi_{\alpha} \otimes \pi_{\beta}$ into $\pi_{\alpha+\beta+2 m}$, proving our theorem.

Using Fourier inversion we have

$$
f(x, y)=(2 \pi)^{-(n-1)} \int_{\mathbb{R}^{2(n-1)}} e^{i\langle x, \xi\rangle+i\langle y, \eta\rangle} \mathcal{F} f(\xi, \eta) d \xi d \eta
$$


We write the differential operator $\mathcal{E}_{\alpha, \beta, m}$ in the proof of Theorem 3.1 as $Q\left(\mathcal{L}_{x}, \mathcal{L}_{y}, \nabla_{x} \cdot \nabla_{y}\right)$ where $Q$ is a homogeneous polynomial of three variables of degree $m$. Thus $\mathcal{D}_{m} f(x)=$ $\left.Q\left(\mathcal{L}_{x}, \mathcal{L}_{y}, \nabla_{x} \cdot \nabla_{y}\right) f(x, y)\right|_{x=y}$. Its action on the inversion formula results in

$$
\begin{aligned}
\mathcal{D}_{m} f(x) & =(2 \pi)^{-(n-1)} \int_{\mathbb{R}^{2(n-1)}} e^{i\langle x, \xi+\eta\rangle} Q\left(-|\xi|^{2},-|\eta|^{2},-\langle\xi, \eta\rangle\right) \mathcal{F} f(\xi, \eta) d \xi d \eta \\
& =(2 \pi)^{-(n-1)} \int_{\mathbb{R}^{n-1}} e^{i(x, \zeta)} \int_{\mathbb{R}^{n-1}} Q\left(-|\zeta-\eta|^{2},-|\eta|^{2},-\langle\zeta-\eta, \eta\rangle\right) \mathcal{F} f(\zeta-\eta, \eta) d \eta d \zeta .
\end{aligned}
$$

That is

$$
\mathcal{F}\left(\mathcal{D}_{m} f\right)(\zeta)=(2 \pi)^{-(n-1)} \int_{\mathbb{R}^{n-1}} Q\left(-|\zeta-\eta|^{2},-|\eta|^{2},-\langle\zeta-\eta, \eta\rangle\right) \mathcal{F} f(\zeta-\eta, \eta) d \eta,
$$

and furthermore

$$
\left|\mathcal{F}\left(\mathcal{D}_{m} f\right)(\zeta)\right|^{2} \leq A(\zeta) \int_{\mathbb{R}^{n-1}}|\mathcal{F} f(\zeta-\eta, \eta)|^{2}|\zeta-\eta|^{2 \widetilde{\alpha}}|\eta|^{2 \widetilde{\beta}} d \eta
$$

with

$$
A(\zeta):=(2 \pi)^{-(n-1)} \int_{\mathbb{R}^{n-1}}\left|Q\left(-|\zeta-\eta|^{2},-|\eta|^{2},-\langle\zeta-\eta, \eta\rangle\right)\right|^{2}|\zeta-\eta|^{-2 \widetilde{\alpha}}|\eta|^{-2 \widetilde{\beta}} d \eta .
$$

To estimate the integral $A(\zeta)$ we write $\zeta=|\zeta| u,|u|=1$, and perform a change of variables $\eta=|\zeta| v$. It is

$$
A(\zeta)=(2 \pi)^{-(n-1)}|\zeta|^{4 m-2 \widetilde{\alpha}-2 \widetilde{\beta}+(n-1)} \int_{\mathbb{R}^{n-1}}\left|Q\left(-|u-v|^{2},-|v|^{2},-\langle u-v, v\rangle\right)\right|^{2}|u-v|^{-2 \widetilde{\alpha}}|v|^{-2 \widetilde{\beta}} d v
$$

and the integral is convergent and uniformly bounded in $u,|u|=1$; indeed it is locally integrable near $v=0$, and $v=u$ for $2 \widetilde{\alpha}, 2 \widetilde{\beta}<n-1$ and is integrable at infinity for the integrand is dominated by

$$
C\left(1+|v|^{2}\right)^{-(\widetilde{\alpha}+\widetilde{\beta}-2 m)}
$$

with $\widetilde{\alpha}+\widetilde{\beta}-2 m=n-1+(n-1-\alpha-\beta-2 m)<n-1$ for some constant $C$. Thus $A(\xi) \leq C|\zeta|^{4 m-2 \widetilde{\alpha}-2 \widetilde{\beta}+(n-1)}$, and

$$
\left|\mathcal{F}\left(\mathcal{D}_{m} f\right)(\zeta)\right|^{2}|\zeta|^{-4 m+2 \widetilde{\alpha}+2 \widetilde{\beta}-n} \leq C \int_{\mathbb{R}^{n-1}}|\mathcal{F} f(\zeta-\eta, \eta)|^{2}|\zeta-\eta|^{2 \widetilde{\alpha}}|\eta|^{2 \widetilde{\beta}} d \eta,
$$

and its integration over $\zeta$ gives

$$
\begin{aligned}
\int_{\mathbb{R}^{n-1}}\left|\mathcal{F}\left(\mathcal{D}_{m} f\right)(\zeta)\right|^{2}|\zeta|^{-4 m+2 \widetilde{\alpha}+2 \widetilde{\beta}-n} d \zeta & \leq C \int_{\mathbb{R}^{n-1}} \int_{\mathbb{R}^{n-1}}|\mathcal{F} f(\zeta-\eta, \eta)|^{2}|\zeta-\eta|^{2 \widetilde{\alpha}}|\eta|^{2 \widetilde{\beta}} d \eta \\
& =C\|f\|_{\alpha \otimes \beta}^{2}
\end{aligned}
$$

whereas the LHS is precisely $\left\|\mathcal{D}_{m} f\right\|_{\alpha+\beta+2 m}^{2}$. This finishes the proof.

When $n=2$ then $m=0$ and the theorem states that $\pi_{\alpha+\beta}$ appears in the tensor product $\pi_{\alpha} \otimes \pi_{\beta}$ if $\alpha+\beta<1$. This has been proved earlier in [24]. 


\section{THE APPEARANCE OF ONE COMPONENT $\pi_{\alpha+\beta}$ IN $\pi_{\alpha} \otimes \pi_{\beta}$ FOR$$
G=S U(n, 1), S p(n, 1)
$$

We treat now the other rank one classical groups. The following result follows straightforward from Theorem 2.1.

Theorem 5.1. Let $G=S U(n, 1)$ and $S p(n, 1), \pi_{\alpha}$ and $\pi_{\beta}$ be the complementary series for $\alpha, \beta$ as in (2.3), $0<\alpha, \beta<\rho=n$ and respectively $2<\alpha, \beta<\rho=2 n-1$. Then the complementary series $\left(\pi_{\alpha+\beta}, G\right)$ of $G$ appears discretely in the tensor product $\pi_{\alpha} \otimes \pi_{\beta}$ if

$$
\alpha+\beta< \begin{cases}n, & \mathbb{F}=\mathbb{C}, \\ 2 n-1, & \mathbb{F}=\mathbb{H} .\end{cases}
$$

Proof. We prove the case for $G=S U(n, 1)$ and the same methods applies also to $G=$ $S p(n, 1)$. We consider the diagonal imbedding of $G$ in $G_{1}=S U(n, 1) \times S U(n, 1)$. It follows from Theorem 2.1 that for $\alpha, \beta \in(0, \rho)$ the complementary series $\pi_{\alpha}$ and $\pi_{\beta}$ appear in $\tau_{\frac{\alpha}{2}}$ and $\tau_{\frac{\beta}{2}}$, respectively. Now $\tau_{\nu}$ of $G_{1}$ is the tensor product $\lambda_{\nu} \otimes \overline{\lambda_{\nu}}$ on $\mathcal{H}_{\nu} \otimes \overline{\mathcal{H}_{\nu}}$ where $\mathcal{H}_{\nu}$ is the space of holomorphic functions on the unit ball $B^{n}$ with the reproducing kernel $(1-\langle z, w\rangle)^{-\nu}$. If $\alpha+\beta<n$ then $\pi_{\alpha}$ appears in $\tau_{\frac{\alpha}{2}}$, so does $\pi_{\beta}$ in $\tau_{\frac{\beta}{2}}$. The tensor product $\tau_{\frac{\alpha}{2}} \otimes \tau_{\frac{\beta}{2}}$ is now

$$
H:=\left(\mathcal{H}_{\frac{\alpha}{2}} \otimes \overline{\mathcal{H}_{\frac{\alpha}{2}}}\right) \otimes\left(\mathcal{H}_{\frac{\beta}{2}} \otimes \overline{\mathcal{H}_{\frac{\beta}{2}}}\right)
$$

Its restriction to $G$ is

$$
H=\left(\mathcal{H}_{\frac{\alpha}{2}} \otimes \mathcal{H}_{\frac{\beta}{2}}\right) \otimes \overline{\left(\mathcal{H}_{\frac{\alpha}{2}} \otimes \mathcal{H}_{\frac{\beta}{2}}\right)} .
$$

However the tensor product $\mathcal{H}_{\frac{\alpha}{2}} \otimes \mathcal{H}_{\frac{\beta}{2}}$ of two holomorphic representation is decomposed discretely under $G$ and contains a component $\mathcal{H}_{\frac{\alpha+\beta}{2}}$; see e. g. [25, 22] and references therein. Thus $H$ contains a discrete component $\mathcal{H}_{\frac{\alpha+\beta}{2}} \otimes \overline{\mathcal{H}_{\frac{\alpha+\beta}{2}}}$. We use again Theorem 2.1 and deduce that this space has a discrete component $\left(\pi_{\alpha+\beta}, G\right)$.

\section{REFERENCES}

[1] C. Asmuth and J. Repka, Tensor products for $\mathrm{SL}_{2}(\mathcal{K})$. I. Complementary series and the special representation, Pacific J. Math. 97 (1981), no. 2, 271-282. MR 641157 (83c:22023)

[2] S. Ben Saïd, K. Koufany and G. Zhang, Invariant trilinear forms on spherical principal series of realrank one semisimple Lie groups, Internat. J. Math. 25 (2014), no. 3, 1450017 (35 pages).

[3] M. Burger, J.-S. Li, and P. Sarnak, Ramanujan duals and automorphic spectrum, Bull. Amer. Math. Soc. (N.S.) 26 (1992), no. 2, 253-257. MR 1118700 (92h:22023)

[4] J.-L. Clerc, Singular conformally invariant trilinear forms and covariant differential operators on the sphere, arXiv:1102.1861.

[5] J.-L. Clerc and R. Beckmann, Singular conformally invariant trilinear forms and generalized RankinCohen operators, arXiv:1104.3461.

[6] J.-L. Clerc and B. Ørsted, Conformally invariant trilinear forms on the sphere, arXiv:1001.2851

[7] L. Clozel, Spectral theory of automorphic forms, Automorphic forms and applications, IAS/Park City Math. Ser., vol. 12, Amer. Math. Soc., Providence, RI, 2007, pp. 43-93. MR 2331344 (2008i:11069)

[8] A. Connes and H. Moscovici, Rankin-Cohen brackets and the Hopf algebra of transverse geometry, Mosc. Math. J. 4 (2004), no. 1, 111-130. 
[9] M. Cowling, A. Dooley, A. Korányi, and F. Ricci, An approach to symmetric spaces of rank one via groups of Heisenberg type, J. Geom. Anal. 8 (1998), no. 2, 199-237.

[10] S. Helgason, Groups and geometric analysis, Academic Press, New York, London, 1984.

[11] B. Ørsted J.-L. Clerc, T. Kobayashi and M. Pevzner, Generalized Bernstein-Reznikov integrals, Math. Ann. 349 (2011), 395-431.

[12] G. van Dijk and S. C. Hille, Canonical representations related to hyperbolic spaces, J. Funct. Anal., 147 (1997), 109-139.

[13] J. Faraut and A. Koranyi, Function spaces and reproducing kernels on bounded symmetric domains, J. Funct. Anal. 88 (1990), 64-89.

[14] K. D. Johnson and N. R. Wallach, Composition series and intertwining operators for the spherical principal series. I, Trans. Amer. Math. Soc. 229 (1977), 137-173. MR MR0447483 (56 \#5794)

[15] A. Knapp, Representation theory of semisimple groups, Princeton University Press, Princeton, New Jersey, 1986.

[16] T. Kobayashi and M. Pevzner, Rankin-Cohen Operators for Symmetric Pairs, preprint, arXiv:1301.2111.

[17] V. F. Molchanov, Canonical representations and overgroups, Lie groups and symmetric spaces, 213224, Amer. Math. Soc. Transl. Ser. 2, 210, Adv. Math. Sci., 54, Amer. Math. Soc., Providence, RI, 2003.

[18] J. Möllers and Y. Oshima, Restriction of most degenerate representations of $O(1, N)$ with respect to symmetric pairs, J. Math. Sci. Univ. Tokyo 22 (2015), no. 1, 279-338.

[19] M. A. Naimark, Decomposition of a tensor product of irreducible representations of the proper Lorentz group into irreducible representations. III, Trudy Moskov. Mat. Obšč. 10 (1961), 181-216. MR 0148800 (26\#6304b)

[20] Yu. A. Neretin and G. I. Ol'shanskii, Boundary values of holomorphic functions, singular unitary representations of the groups $\mathrm{O}(p, q)$ and their limits as $q \rightarrow \infty$, J. Math. Sci. (New York) 87 (1997), no. 6,39834035

[21] V. Ovsienko and P. Redou, Generalized transvectants-Rankin-Cohen brackets, Lett. Math. Phys. 63 (2003), no. 1, 19-28. MR 1967533 (2004c:58077)

[22] L. Peng and G. Zhang, Tensor products of holomorphic representations and bilinear differential operators, J. Funct. Anal. 210 (2004), no. 1, 171-192.

[23] L. Pukánszky, On the Kronecker products of irreducible representations of the $2 \times 2$ real unimodular group. I, Trans. Amer. Math. Soc. 100 (1961), 116-152. MR 0172962 (30 \#3177)

[24] J. Repka, Tensor products of unitary representations of $S L_{2}(\mathbf{R})$, Amer. J Math. 100 (1978), 930-932.

[25] J. Repka, Tensor products of holomorphic discrete series representations, Can. J. Math. 31 (1979), 836-844.

[26] B. Speh and T. N. Venkataramana, Discrete components of some complementary series representations, Indian J. Pure Appl. Math. 41 (2010), no. 1, 145-151. MR 2650105 (2011h:22014)

[27] B. Speh and G. Zhang, Restriction to symmetric subgroups of unitary representations of rank one semisimple Lie groups, Math. Zeit. 283, 629-647.

[28] D. A. Vogan, Jr. and N. R. Wallach, Intertwining operators for real reductive groups, Adv. Math. 82 (1990), no. 2, 203-243. MR 1063958 (91h:22022)

[29] G. Zhang, Discrete components in restriction of unitary representations of rank one semisimple Lie groups, J. Funct. Anal. 269 (2015), no. 12, 3689-3713.

Mathematical Sciences, Chalmers University of Technology and Mathematical SciENCES, GÖTEBorg UNIVERSITY, SE-412 96 GÖTEBorg, SWEDEN

E-mail address: genkai@chalmers.se 